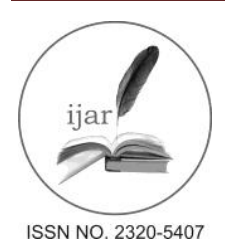

\author{
Journal homepage: http://www.journalijar.com

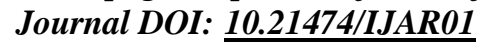

INTERNATIONAL JOURNAL

OF ADVANCED RESEARCH

RESEARCH ARTICLE

\title{
ON UNEXPECTED DIFFICULTIES IN THE TEACHING OF THE SPECIAL THEORY OF RELATIVITY.
}

\author{
*Andrew Chubykalo ${ }^{1}$, Augusto Espinoza ${ }^{1}$, and Sergey Artekha ${ }^{2}$. \\ 1. Unidad Académica de Física, Universidad Autónoma de Zacatecas, Zacatecas, México \\ 2. Space Research Institute of RAS, Moscow, Russia.
}

\section{Manuscript Info}

Manuscript History:

Received: 12 May 2016

Final Accepted: 19 June 2016

Published Online: July 2016

Key words:

Time dilation,

Length contraction,

Lorentz transformations,

Transverse Doppler effect.

*Corresponding Author

\section{Abstract}

The wide availability of information has led to the fact that students are well aware of the difficulties of existing physical theories. In this article we analyze some logical paradoxes of the special relativity theory (SRT), concerning the time dilation and length contraction. The Lorentz transformations and the relativistic law for velocity addition are considered in detail. The notion of relativity and the transverse Doppler effect are discussed. The whole complex of numerous paradoxes in the special theory of relativity worries us by further complications.

Andrew Chubykalo.

Copy Right, IJAR, 2016,. All rights reserved.

\section{Introduction:-}

Albert Einstein begins his famous paper (Einstein, 1905) with the critic of non-symmetricity of Maxwell's equations. But these equations represent a generalization of experimental physical laws and observations. Later, Einstein rejected the Abraham force only on the grounds that it is not deduced from the general theoretical principles, though, gives a much better agreement with experiments than a formula of the special relativity theory (SRT). Such statements are counterproductive from an educational point of view. Based on some of Einstein's memories, the opinion in (Frankfurt et al., 1972) suggests that the creation of the SRT - is a purely theoretical "breakthrough" and has no need in the experimental studies at all. Can some mathematized theoretical principles be valued higher than experiments in the natural sciences? Obviously not! And students are well aware of the distinction between subjects of physics and mathematics.

The kind of "asymmetry" of mathematical arguments is well-known: an infinite number of confirming examples cannot outweigh even one counterexample (the logical contradiction). Logic, as part of common sense, is more than any particular theory: all sciences are based on it, and logically-inconsistent theory cannot be considered as scientific one. Students constantly test the strength of scientific theories with difficult questions. Students constantly test the strength of scientific theories with unexpected difficult questions. The SRT also cannot remain an exception for the constructive logical analysis in the process of teaching.

In the teaching of the SRT in the general physics course, as a rule, one uses mental experiments with two "objects" (the pairwise synchronization). In this case there exists a unique mathematical relationship between classical concepts and relativistic ones (the recalculation is possible). This fact was perfectly understood by Henri Poincare. He considered new concepts and transformations as only one of the possible "agreements" on a par with the previous classical concepts (see in (Poincare, 1983) the work "Final Thoughts", chapter II and the commentary at the end of the book - the article of M.I. Panova, A.A. Tyapkin and A.S. Shibanova). In the theoretical physics course, however, relativistic concepts often brings difficulties when describing the spatial movement (not along one and the same line) and when the number of objects is greater than two. 
Recently, multiple logical paradoxes and inconsistencies of the SRT increasingly attract the attention of professionals and are subjected to legitimate criticism (Akimov, 2001; Smulsky, 2004; List of some antirelativistic works (1905-2003)). Students discover that though each element of the SRT is non-contradictive locally, but the complete construction can contain a contradiction.

The present paper discusses new paradoxes of the SRT and some aspects of this theory, dealt with in the course of teaching of the SRT.

\section{On complexities in teaching of SRT:-}

The theory of relativity has problems with the declared properties of the homogeneity and isotropy of space and physical meaning of used values. Let us start with a discussion of some paradoxes.

We traditionally begin with the paradox of twins: to explain it, the acceleration of one of the twins is involved. However, only manipulations with "mathematical letters" are not enough for modern students. Physicists search for the causes of phenomena, mechanisms for their implementation and the physical meaning of the used values. This is just what physics is different from mathematics. According to the SRT we shall remind ourselves that before acceleration, in opinion of each brother, the other one should appear younger. Since the rejuvenation is impossible, the twin-astronaut cannot became younger after acceleration, but the twin-homebody became much older. Since the only influence was acceleration, then, from the viewpoint of the twin-astronaut, he is accelerated, but the other brother grows older! Where is the cause of the phenomenon presented here? And there is no mechanism! Further, we can see from Fig. 1 that the path length $|O A|$ and $|B C|$ with acceleration (for example, with the usual $g$ ) can be chosen to be the same one (fixed). But for different cases, we can change the path length $|A B|$ of flight with a constant (on the module) big speed. For example, we can choose the distance of 100 light years in the first case and of 1000 light years in the second case.

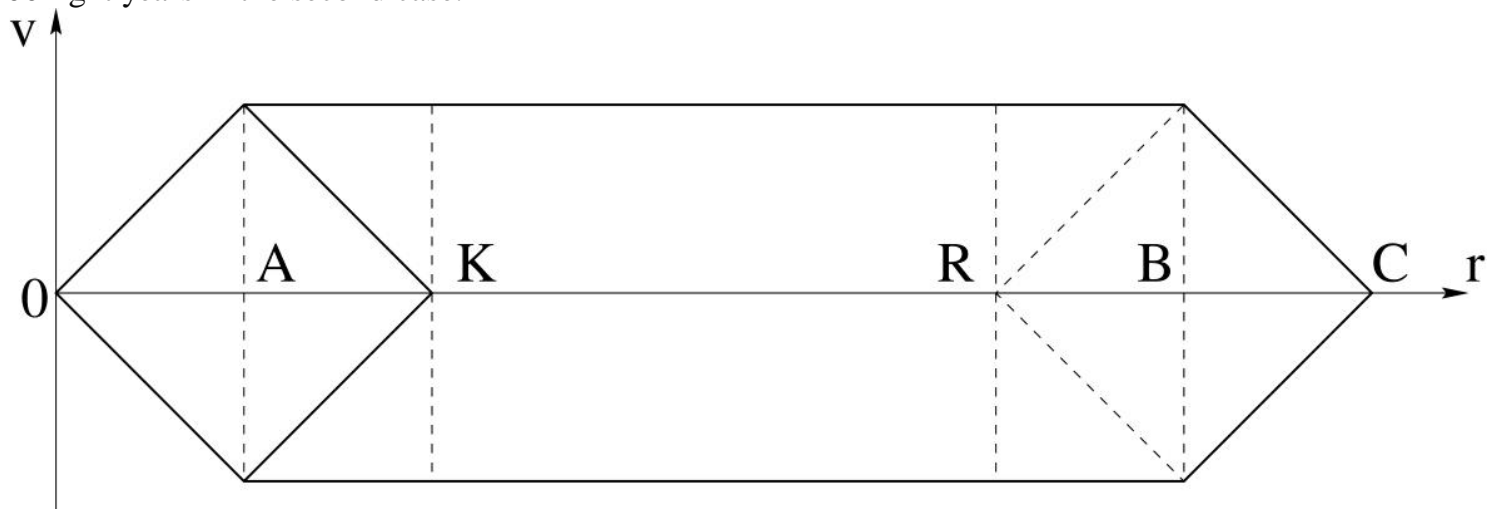

Fig. 1:- The "role" of accelerations in the paradox of twins.

It is clear that the same acceleration cannot explain differences in the age of the twins (100 or 1000 years) for these different cases. Otherwise the causality is lost: the acceleration is just one and the same! Moreover, the brotherhomebody cannot be too lazy and "take part" only in the accelerated movements at sections $|\mathrm{OA}|$ and $|\mathrm{AK}|$, which are completely identical to the analogous sections for the brother-astronaut. Starting at the calculated moment when the brother-astronaut will fly through point $\mathrm{R}$, we see only the displacement of starting time of accelerated motions. The differences in the accelerations disappear. Thus, the initial explanation of the twin's paradox by means of acceleration, to which adhered Einstein, Pauli, Born and others, is not convincing to modern students.

Students are accustomed that in classical physics, results obtained by one observer can be used by any other observer (including investigators not participating in experiments). In such a case, one can formulate some symmetric setting of a problem with results which are evident from common sense. But, from the SRT viewpoint, one must consider different results from the viewpoint of different observers and compare all results between themselves. Let two colonies of Earth's inhabitants A and B be at some large distance from each other (Fig. 2). A beacon $\mathrm{O}$ is at the middle of this distance. It sends a signal (the light sphere), and when it reaches both colonies 
(simultaneously), each launches a spacecraft with families of astronauts. The laws of acceleration (to reach large equal speeds) are chosen equal in advance.

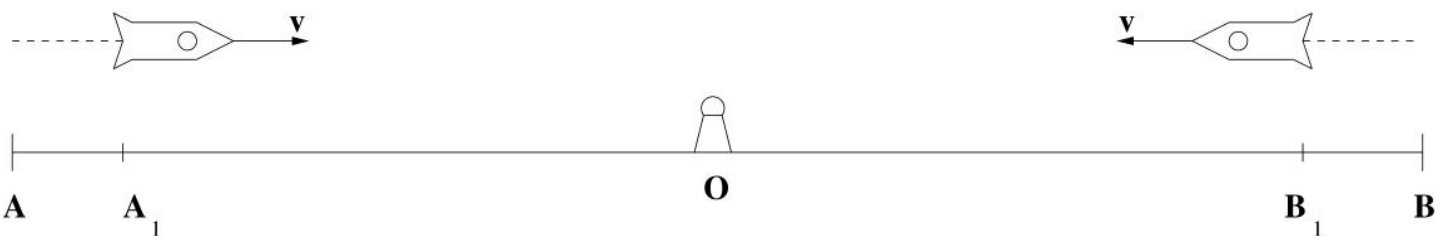

Fig. 2:- The paradox of coevals.

We formulate a paradox of coevals - people born in the same year. (In SRT a change of time course is declared rather than a transfer of initial time, as the time zone on the Earth, for example.) Babies are born on each spacecraft just after accelerations became equal to zero. And these babies are chosen for a comparison of age. All previous history of motion (up to the points $A_{1}$ and $B_{1}$ respectively) does not exist for them. The stationary observers at the points $A_{1}$ and $B_{1}$ can confirm the fact of the births of the babies. The babies differ in that they moved relative to each other at a certain speed all the time. They travelled equal distances $\left|\mathrm{OA}_{1}\right|=\left|\mathrm{OB}_{1}\right|$ up to the meeting. For example, let the flight of baby 1 with the constant speed takes place for a time of 15 years. From the SRT viewpoint, the first baby will reason in the following manner: the second baby moved relative to me with a large velocity all my life (15 years); therefore, his age must be less than mine. Besides, if he counts out the age of the second baby starting from the moment of the receipt of the signal from $\mathrm{B}_{1}$, then he will believe that he will see an infant with his feeding bottle at the meeting. But the second baby will reason about the first baby in the same manner. However, owing to the full symmetry of the motion, the result is obvious: the age of both "astronauts" are the same (this fact will be confirmed by the stationary observer at the beacon). The astronauts can photograph themselves at this instant and write their age on the back side of the picture (or even to exchange pictures by a digital method). It is nonsense, if wrinkles appear on the face in the picture of any of the astronauts during the deceleration of the other one. Besides, it is unknown beforehand if one of the astronauts will wish to move with acceleration in order to turn around and catch up with the other one. How to explain these modified paradoxes to students?

The next paradox is also not clear to the students. Imagine that two identical spaceships fly along two identical circular orbits, one nearby the other (or even in the bound state) around a star. Obviously, according to the SRT (and to the general relativity theory - GRT) time flows equally on both systems fixed relative to each other's spaceship. Consider now the second situation (Fig. 3) - we split the spaceships and turn one of the orbits around an arbitrary diameter on 180 degrees. Now spaceships move along the same orbits, with the same speed but rotate around the star in opposite directions, meeting twice during one revolution (at the points A and B).

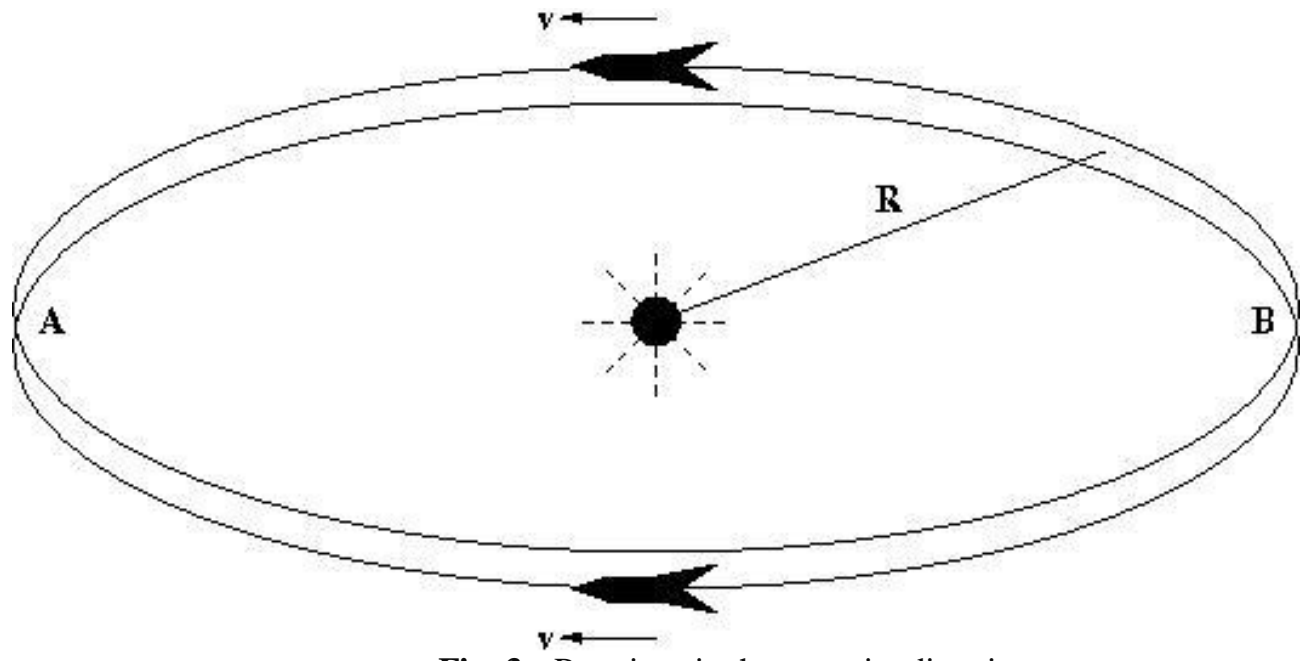

Fig. 3:- Rotations in the opposite directions. 
It is obvious that the influence of the GRT effects on the course of time for both spaceships remained unchanged. But there is the contradiction with the effect of SRT - now spaceships move relative to each other with a non-zero speed all the time (recall that the relativistic formula includes the square of the speed). Not nanoseconds, but rejuvenating apples would be painted here! Since the number of revolutions can be arbitrary, it remains only to decide this, to whom of them award the "prize": to being younger? To whom moves clockwise or counterclockwise? And from which side it is necessary to see - from above or below? Actually, it is obvious for students that the problem is completely symmetrical, and no difference in time can exist. Does this mean that the contribution from the SRT effects (relativistic time dilation) is completely absent? The centrifugal acceleration (noninertiality) prevents relativism? No problem! Students can easily find the limit values. Let the linear velocity of the spaceships be close to the speed of light. We will increase the radius of the orbit $R$ so that the value of $c^{2} / R$ tends to zero (for example, there were many orders of magnitude smaller than the existing accuracy of its measurement). Then no experiments detect the non-inertiality. The ratio of centrifugal acceleration to the centrifugal acceleration on the Earth can be made less than any arbitrarily small value $\varepsilon$ by choosing large radius R. For example, you can take $\varepsilon \sim 10^{-10}$ or $\varepsilon \sim 10^{-100}$. But all SRT experiments performed on the Earth with $\varepsilon \sim 1$ ! As is clear for students, there is no sense to fight for the need of strict inertiality; otherwise there would be no subject of study itself for SRT at all.

We can construct a symmetrical scheme of flower-type (Fig. 4), which includes rectilinear sections, where a large speed remains constant (inertial systems). The movement of each spaceship consists of 5 segments (see the separate enlarged i-th trajectory on the right).

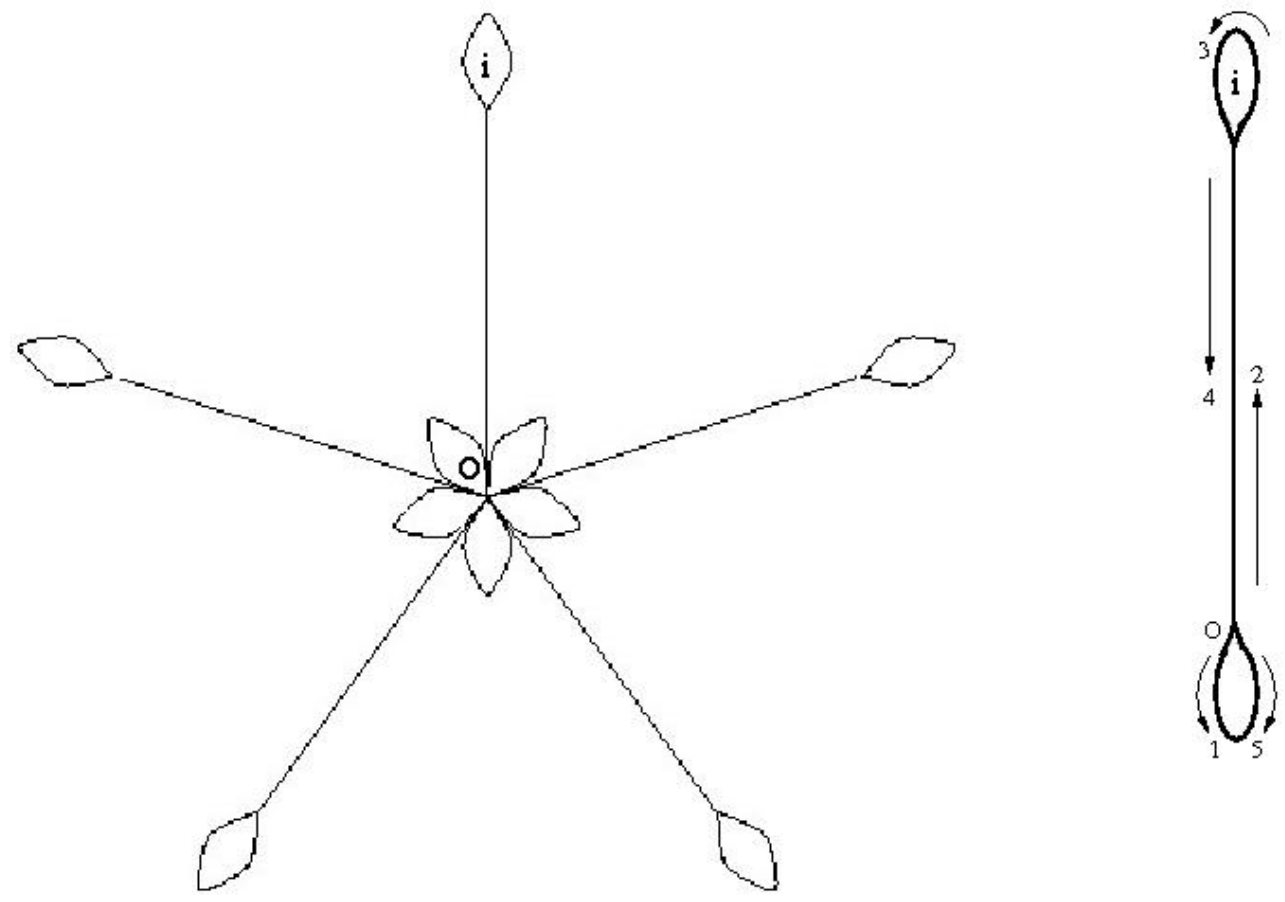

Fig. 4:- Flyings over the symmetrical scheme of flower-type.

Starting from one point with unified acceleration (for example, g) along identical loops (accelerating segments 1), spaceships pass again the same single point $O$. Therefore, the time spent by each spaceship (i or $j$ ) on this speedingup will be the same both from the viewpoint of a quiescent observer and from the viewpoint of any astronaut: $t_{1 i}=t_{1 j}$. Further, the spaceships move uniformly and rectilinearly (inertial path sections 2 ). After that identical rotary loops (segments 3 ) follow. For the rotary loops, it is also easy to prove that $t_{3 i}=t_{3 j}$ (any loop can be obtained from another one with the help of some parallel translation and some turn). Further, the spaceships again move uniformly and rectilinearly (inertial sections 4 coincide with inertial sections 2 , but in reverse order). Finally, movements are completed in brake loops (path sections 5 coincide with path sections 1). Deceleration is performed in the reverse order to the starting acceleration: $t_{5 i}=t_{5 j}=t_{1 i}=t_{1 j}$. The scheme is completely symmetrical, so the 
total time of the motion for all the astronauts is one and the same: $t_{i}=t_{j}$. Everything said above reflects the properties of isotropy and homogeneity of space. Since the motion of each spaceship consists of motions along these five path sections, we finally have for the rectilinear path sections (inertial sections): $t_{2 i}+t_{4 i}=t_{2 j}+t_{4 j}$ for any $i$ and $\mathrm{j}$. However velocity has vector nature, and the relative velocity depends on $\mathrm{i}$ and $\mathrm{j}$. And the SRT formula includes the square of the relative velocity only. Hence, according to the SRT the course of time must be different, and that leads to contradictions both between the astronauts and with the data of the stationary observer. Thus, the presence of some non-zero relative velocity cannot be the cause for the effect of the time dilation. Students easily come up with such symmetric schemes.

Note some strange thing concerning reversibility. In transition from one inertial frame of reference to the other and back, the linear Lorentz transformations are fully equivalent both for coordinates and for the time (they are reversible). Then it seems strange for students that a difference between lengths of bodies vanishes with the return to the initial place in the SRT (for example, in the paradox of twins), but the disparity remains in the time elapsed.

A methodically correct aspect of classical physics is the comparison of the running of an arbitrary process with a standard one, i.e. with process completely independent of it. This allows you to compare different processes with each other for a unified objective description of reality. The relativistic method is strange for students (like using our own heartbeats in measuring the time, or pigeon post for the synchronization method). The infinitely remote source of periodic signals, which is situated perpendicular to the direction of the body motion (the relative motion of bodies or systems), can serve as a watch counting the universal absolute time (which remains one and the same regardless of choice of the inertial system of reference).

Now we consider some disputable points in relativistic spatial concepts. Imagine that three spaceships with astronauts flew in the direction to the coordinate origin. One spaceship moved uniformly along the X-axis for 100 years with a speed 0.99c, the second spaceship moved uniformly along the Y-axis for 1000 years with a speed $0.9999 \mathrm{c}$ and the third spaceship moved uniformly along the Z-axis for 1 million years with a speed 0.999999c. And these three spaceships simultaneously pass the origin of coordinate. All the astronauts look at the surrounding Universe and make an exchange by telegrams. Astronauts from the first spaceship argue that the whole Universe is reduced along the $\mathrm{X}$-axis by 10 times, while astronauts from the second spaceship believe that the Universe only shrank along the Y-axis by 100 times, while astronauts from the third spaceship are convinced that the same Universe shrank by 1000 times along the Z-axis. How to explain to the students that the movement of a spaceship compresses the entire universe without any physical mechanism? Or the entire universe is not compressed, but only a part (respectively of 100, of 1000 and of 1000000 light years), not to violate the causality principle? And there appears a gap with the rest of the Universe? Any of the choices is an obvious nonsense!

Pay attention to another strangeness (the paradox of distances). Since the shortening of lengths of objects is associated with properties of space itself, the distance to objects must also be shortened (regardless of whether we approach the object or move away from it). Therefore, if the speed of a spaceship is high enough $(\mathrm{v} \rightarrow \mathrm{c})$, we cannot only look at distant stars, but also "touch" them, because our own dimensions do not change in our own reference system. Besides, when flying away from the Earth for a long time (the value of acceleration is not limited by SRT), we will eventually be at the distance of just "one meter" from it. At which time instant will the observer at this distance in "one meter", see the reverse motion of the spaceship (contrary to the action of rocket engines)? Now we consider the relativistic effect of contraction of distances (the paradox of pedestrians). We will "agree in advance" about the following mental experiment (Fig. 5).

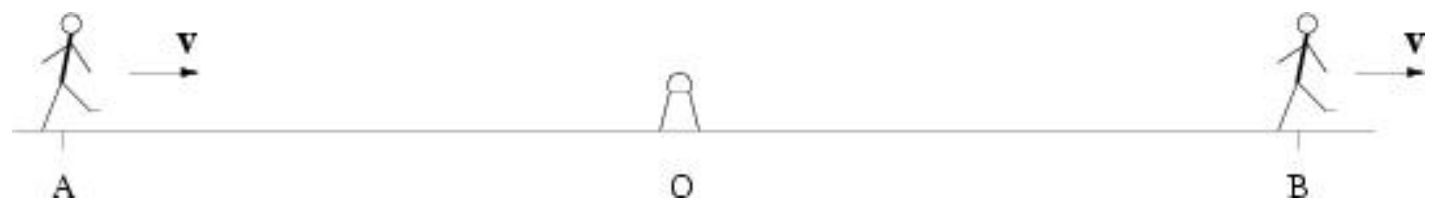

Fig. 5:- The paradox of two pedestrians.

Let a beacon placed at the middle of a segment send a signal toward its ends. Let the length of the segments be one million light years. At the time of arrival of a flash: two pedestrians at the ends of the segments begin to go at equal 
speed towards a single preselected side, along the straight line containing the given segment, and they will walk for several seconds. The moving segment (a system of two pedestrians) should be shortened relative to the ends of the motionless segment by some hundreds of kilometers. However, none of the pedestrians will "fly away" for hundreds of kilometers during these seconds. The moving segment cannot either be torn off at the middle because the Lorentz transformation laws are continuous. So, where has this segment been shortened in such a case? And how can this be detected? Observability of relativistic kinematic effects is always doubtful for students.

Let us recollect the method of division of the whole into parts in the Galilee proof that there are no reasons to increase twice the acceleration of free falling with the increasing twice of the mass of a falling body. Let us consider now the paradox of a cut-in-half ruler. Four identical rulers are represented in Fig. 6:

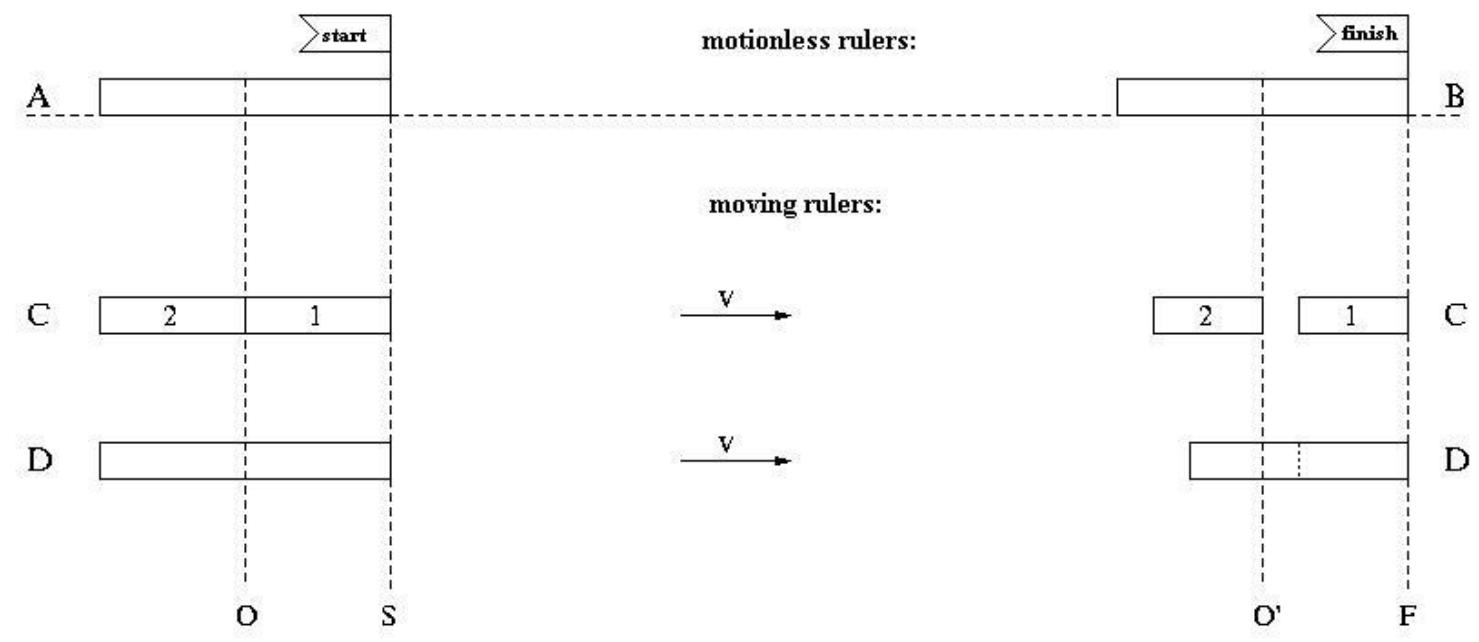

Fig. 6:- The paradox of a sawn ruler.

Ruler A lies motionlessly on start and ruler B lies motionlessly on finish as the standards (only for presentation). Ruler $\mathrm{C}$ will move during the experiment being cut on two equal parts 1 and 2 . But ruler D will move as the whole during experiment. We separately consider the movement of the first half of ruler $\mathrm{C}-1$. It starts to move with uniform acceleration, reaches the big speed $\mathrm{V}$, flies with such a constant speed and crosses the final straight $\mathrm{F}$ by the right end. If the second half of ruler $\mathrm{C}-2$ started to move simultaneously with the first half $\mathrm{C}-1$ and moved under the same law (as the first half $\mathrm{C}-1$ ), then its side will cross line $\mathrm{O}^{\prime}$ at the time of crossing of the finish by the first half of a ruler $\mathrm{C}-1$. This result is obvious, since the situation with the second half of a ruler differs from the situation with the first half of a ruler only by parallel translation of the beginning of coordinates (the right end of a half of a ruler from line $\mathrm{S}$ to line 0 ). However, for the uncut ruler $\mathrm{D}$ the situation on the finish will be quite different (the ruler reached the finish as the whole one). We have a logic contradiction. First, whence can ruler $\mathrm{C}$ know about its cutting? Secondly, the cut of the zero value cannot turn to a nonzero spatial gap according to the SRT. Besides, a ruler can be cut in 3 and more parts and it is impossible to rescue all cuts from the gap. How can we explain to students the use of parallel translation in SRT?

Now we consider the spatial paradox connected with ostensibly existing relativistic turn of a rod. A thin rod of some length $\mathrm{L}$ flies along the $\mathrm{X}$-axis with a speed $\mathrm{v}$, and the plate with a niche of the same size $\mathrm{L}$ runs with a speed $\mathrm{V}$ in a direction of the Z-axis, so that in the classical case the rod will precisely pass through the niche. Contradictions are ostensibly eliminated in indications of different observers by the introduction of the relativistic turn of the rod. However, the situation with the relativistic angle of the rod turning elementarily becomes a dramatic one, since it uniquely depends on the ratio of speeds. Let the other smaller rod l slide with some speed on our rod. Observers on both rods will claim that the clearance between the rods is absent. However, according to the SRT (due to a different speed of rods $\mathrm{v}$ and $\mathrm{v}_{1}$ ), the big rod $\mathrm{L}$ and the small rod $\mathrm{l}$ should be turned at different angles relative to the plate for the observer on the plate. That is in the SRT the small rod will be turned upwards relatively to the big rod, and there appears a clearance between the rods. We have the obvious contradiction. 


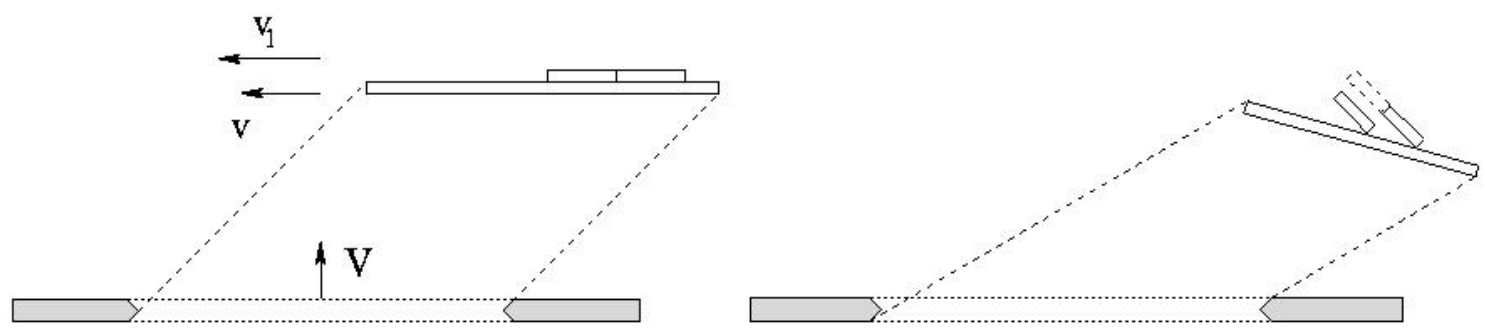

Fig. 7:- The paradox of sliding rods.

This contradiction becomes even more vivid if we use the principle of division of a whole into pieces. Then if we consider rod l as a single whole, one situation (see Fig. 7) turns out, as if the second half of rod l is raised at some height above rod L, on which a sliding takes place. But if we consider the small rod consisting of two real halves, then the given situation for the second half-rod is simply similar to the case of translation of origin and these halves appear with the forward ends on the big rod, but spatially divided (see Fig. 7). The last situation is especially strange, since the cut of the zero size should remain zero size at any turns or multiplications on the relativistic factor. Let us notice that we "tried to help" the SRT a little more, rotating the small half-rods ABOVE the greater one. Actually, in the SRT there are no real firm bodies at all, impenetrable one to the other. All SRT formulas are derived for light flashes, but they are capable to pass through each other. As a result, to reconcile evidences of arbitrary observers (for example, at the rod center), it is necessary to assume as if one rod passes through another. Students descry such the difficulties.

In the course of general physics, the relativistic law for velocity addition is usually considered in the onedimensional case. For two systems participating in relative motion, the determination of their relative velocity causes no doubts (neither in classical physics nor in SRT). Let system $S_{2}$ be moving relative to system $S_{1}$ at speed $v_{12}$; similarly, let system $S_{3}$ be moving relative to $S_{1}$ in the same direction at speed $v_{13}$. In fact, the relativistic law for velocity addition defines the relative speed of that motion in which the observer does not participate: the speed of motion of system $S_{3}$ relative to $S_{2}$ is determined as

$$
\mathrm{v}_{23}=\frac{\mathrm{v}_{13}-\mathrm{v}_{12}}{1-\mathrm{v}_{13} \mathrm{v}_{12} / \mathrm{c}^{2}}
$$

It is precisely this form (although usually $v_{13}$ is expressed in terms of $v_{12}$ and $v_{23}$ ), which discloses the real essence of this law: it tells what relative speed of systems $S_{3}$ and $S_{2}$ will be recorded by the observer in $S_{1}$, if the Einstein light-signal method is used for time synchronization and for measuring length.

Consider the following methodological remark. One rather strange fact from the SRT is the non-commutativity of the relativistic law for velocity addition of non-collinear vectors. The property of non-commutativity and the fact, that the Lorentz transformations without rotations do not compose a group, are mentioned in some theoretical physics textbooks. By contrast, a similar property in quantum mechanics essentially changes the entire mathematical formalism and physically expresses a simultaneous immeasurability of non-commutating values. What fundamentally changed in the later case? How to justify the connection of physical quantities and the used mathematical apparatus for students and postgraduates?

It is seen from the general relativistic law of addition of velocities that

$$
\mathbf{v}_{3}=\frac{\left(\mathbf{v}_{1} \mathbf{v}_{2}\right) \mathbf{v}_{1} / \mathbf{v}_{1}^{2}+\mathbf{v}_{1}+\sqrt{1-v_{1}^{2} / c^{2}}\left(\mathbf{v}_{2}-\left(\mathbf{v}_{1} \mathbf{v}_{2}\right) \mathbf{v}_{1} / v_{1}^{2}\right)}{1+\left(\mathbf{v}_{1} \mathbf{v}_{2}\right) / c^{2}}
$$

Clearly, the result depends on the order of transformation. For example, in the case of sequence $+v_{1} \mathbf{i},-v_{1} \mathbf{i},+v_{2} \mathbf{j},-v_{2} \mathbf{j}$, where $\mathbf{i}, \mathbf{j}$ are the unit vectors of the Cartesian coordinate system, we obtain the zero total velocity. But for the other order of the same quantities $+v_{1} \mathbf{i},+v_{2} \mathbf{j},-v_{1} \mathbf{i}, v_{2} \mathbf{j},-v_{2} \mathbf{j}$ we obtain the non-zero total 
velocity, which depends on $v_{1}$ and $v_{2}$ in a rather complicated manner. The successive application of transformations (of motions) of $\mathrm{v}_{1} \mathbf{i}$ and $\mathrm{v}_{2} \mathbf{j}$ gives

$$
\mathbf{v}_{3}=\mathrm{v}_{1} \mathbf{i}+\sqrt{1-\mathrm{v}_{1}^{2} / \mathrm{c}^{2}} \mathrm{v}_{2} \mathbf{j}
$$

But in the other order of $\mathrm{v}_{2} \mathbf{j}$ and $\mathrm{v}_{1} \mathbf{i}$ it gives

$$
\mathbf{v}_{3}^{\prime}=\mathrm{v}_{2} \mathbf{j}+\sqrt{1-\mathrm{v}_{2}^{2} / \mathrm{c}^{2}} \mathrm{v}_{1} \mathbf{i} .
$$

That is, we have different vectors (Fig. 8). In such a case, what can the decomposition of the velocity vector into components mean? And how does this to explain to students?

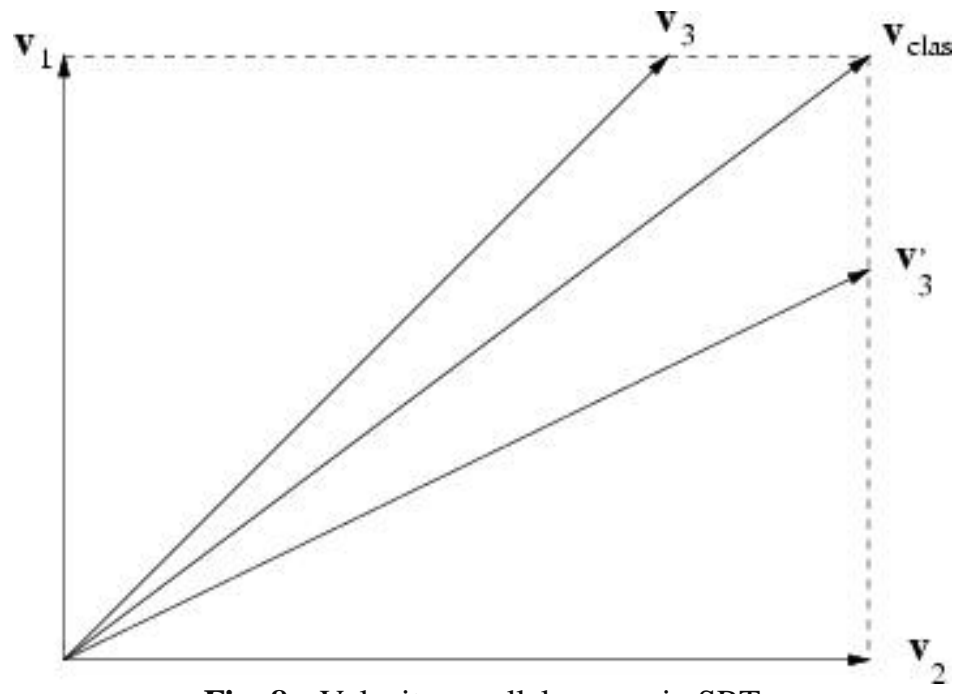

Fig. 8:- Velocity parallelograms in SRT

How to demonstrate to students: can the Lorentz transformation laws describe successive transitions from one inertial system to another, and can the relativistic law of velocity addition correspond to real velocity changes? At first let us recall the meaning of the relativistic law of velocity addition. It must prove that the addition of any motions cannot lead to a speed greater than light speed. For example, the Earth moves relative to stars (factually, it is the first reference system), a spacecraft flies up from the Earth with high velocity (in fact, the second reference system is "created"), then, another spacecraft flies up from the first spacecraft (factually, the third reference system is "created"), and so on. It is just the meaning for consecutive transformations (it is important for non-commutative transformations: which of the velocities considered as the first velocity, which is the second one, etc.). Let us consider now the Lorentz transformation law for arbitrary directions of motion:

$$
\begin{gathered}
\mathbf{r}_{1}=\mathbf{r}+\frac{1}{\mathrm{~V}^{2}}\left(\frac{1}{\sqrt{1-\mathrm{V}^{2} / \mathrm{c}^{2}}}-1\right)(\mathbf{r V}) \mathbf{V}+\frac{\mathbf{V t}}{\sqrt{1-\mathrm{V}^{2} / \mathrm{c}^{2}}}, \\
\mathrm{t}_{1}=\frac{\mathrm{t}+(\mathbf{r V}) / \mathrm{c}^{2}}{\sqrt{1-\mathrm{V}^{2} / \mathrm{c}^{2}}} .
\end{gathered}
$$

It can be easily verified, that the successive application of the relativistic law of velocity addition (2) to quantities

$$
\mathrm{v}_{1} \mathbf{i}, \quad \mathrm{v}_{2} \mathbf{j}, \quad-\mathrm{v}_{1} \mathbf{i}-\sqrt{1-\mathrm{v}_{1}^{2} / \mathrm{c}^{2}} \mathrm{v}_{2} \mathbf{j}
$$


gives zero. To an arbitrary vector $\mathbf{r}=\mathrm{xi}+\mathrm{yj}$ we apply the Lorentz transformation laws successively with the same set of velocities. Then we have:

$$
\begin{aligned}
& r_{1}=\frac{x+v_{1} t}{\sqrt{1-v_{1}^{2} / c^{2}}} i+y j \\
& t_{1}=\frac{t+x v_{1} / c^{2}}{\sqrt{1-v_{1}^{2} / c^{2}}}
\end{aligned}
$$

Further, we have:

$$
\begin{gathered}
\mathbf{r}_{2}=\frac{\mathrm{x}+\mathrm{v}_{1} \mathrm{t}}{\sqrt{1-\mathrm{v}_{1}^{2} / \mathrm{c}^{2}}} \mathbf{i}+\frac{\mathrm{y} \sqrt{1-\mathrm{v}_{1}^{2} / \mathrm{c}^{2}}+\mathrm{v}_{2} \mathrm{t}+\mathrm{xv}_{1} \mathrm{v}_{2} / \mathrm{c}^{2}}{\sqrt{1-\mathrm{v}_{1}^{2} / \mathrm{c}^{2}} \sqrt{1-\mathrm{v}_{2}^{2} / \mathrm{c}^{2}}} \mathbf{j} \\
\mathrm{t}_{2}=\frac{\mathrm{t}+\mathrm{xv}_{1} / \mathrm{c}^{2}+\mathrm{yv}_{2} \sqrt{1-\mathrm{v}_{1}^{2} / \mathrm{c}^{2}} / \mathrm{c}^{2}}{\sqrt{1-\mathrm{v}_{1}^{2} / \mathrm{c}^{2}} \sqrt{1-\mathrm{v}_{2}^{2} / \mathrm{c}^{2}}}
\end{gathered}
$$

We will not write down the expressions for $\mathbf{r}_{3}$ and $\mathrm{dt}_{3}$ in the explicit form because of their awkwardness. However, students can use graphical programs and can be assured of the following properties:

1) In the new system, the initial time is desynchronized at any point of space except the coordinate origin.

2) The time intervals have changed: $t_{3} \neq d t$; that is, we found ourselves in a new moving system, rather than in the initial stationary system.

3) Line segments became not only of changed length, but also are rotated at some angle. We can easily be convinced of this if we find numerically the angle of rotation:

$$
\alpha=\arctan \left(\frac{y_{3}[x(1), y(1), t]-y_{3}[x(0), y(0), t]}{x_{3}[x(1), y(1), t]-x_{3}[x(0), y(0), t]}\right)-\arctan \left(\frac{y(1)-y(0)}{x(1)-x(0)}\right) .
$$

Physically this situation is difficult for students. How it can be proved the objective character of the Lorentz transformation laws and of the relativistic law of velocity addition, if students see the disagreement with each other? Since we have successively passed from one inertial system to another, but the rotation implies the non-inertial character of a system, the SRT itself escapes the limits of its own applicability. If this rotation were real, this would imply a non-objective character of the notion of "inertial system" (since the result would depend on the method of transition to the given system) and, as a consequence, the lack of a proper basis for the existence of the SRT itself. How then to explain to students the concept of inertial system?

The transformation of forces in the SRT looks methodically unclear at transition from the one frame of reference to another. Let us consider, for example, two identical in absolute value charges $+e$ and $-\mathrm{e}$ being at distance $r$ from each other (Fig. 9). 


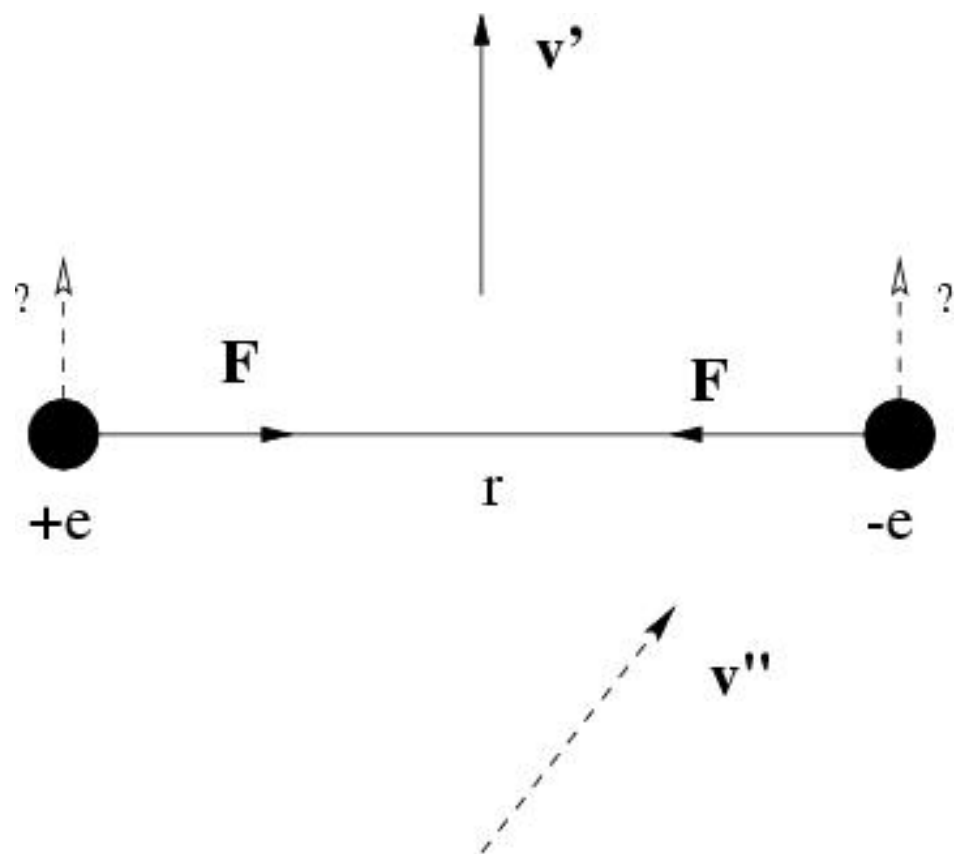

Fig. 9:- Parallel flying charges

In the frame of reference bound with resting charges there exists the electric force $F=e^{2} / r^{2}$ acting between the charges. Look now at the same charges from the system moving at velocity $\mathbf{v}^{\prime}$ perpendicular to the line connecting the charges (in this system the charges are flying parallel to each other). According to the SRT, now the force acts between the charges: $F^{\prime}=G^{2} / r^{2}$, where $G=\sqrt{1-v^{\prime} 2 / c^{2}}$. To what physical quantity should the transformation factor $\mathrm{G}$ be related? The charge is invariant in the SRT. Distance $r$, which is perpendicular to the motion, does not change either. How to explain to students the physical reasons for the change of forces? Note one more strange thing: if the velocity of a observer $\mathbf{v}^{\prime \prime}$ has a component along the line which connects the charges, the force acting on the charges has a component which is perpendicular to this line (i.e. the picture of motion is essentially changed). The complexity for the students is the idea that the one and the same force can be different for different systems of observation. Really, the way of writing Arabic cipher on a dynamometer is independent on observer motion, i.e. readings of the dynamometer (fixing the force) will not be changed with observer motions. Any force acts between the "source" of this force and the concrete "object" of the applied force. What is the relationship of all this with the motion of some "strange eyes"? That is, the force can depend on the source properties, object properties, and their mutual motion.

Of course, a finite time for propagation and transmission of interactions results in a change of the observed motion of particles. An additional dependence of quantities on velocity appears; for example, for an effective mass (more precisely for the effective force). This can be understood qualitatively from the following elementary mechanical model. Consider just one-dimensional motion; let a source emit continuously and uniformly similar particles flying at a constant speed $\mathrm{v}_{1}$ along the straight line. At any place of the straight line, a test body placed to rest will be subject to action of a constant pressure force (from bombarding particles). If now a test body moves away from the source at some velocity $\mathrm{v}$, then the number of particles reaching this body per time unit will decrease. This can be interpreted as a decrease of the effective force (or seeming increase of the effective mass). Being accelerated under the effect of particles in the limit $\mathrm{v} \rightarrow \mathrm{v}_{1}$, the seeming effective mass of the body tends to infinity (more correctly to say, the effective force tends to zero).

The relativistic equation of movement with a force $\mathbf{F}$ can be elementarily written down as the classical second law of Newton with some other force $\mathbf{F}^{\prime}$. For this purpose it is necessary to find a derivative explicitly on the left-hand side of the relativistic equation $\mathbf{d p} / \mathrm{dt}=\mathbf{F}$ and to multiply in scalar way the left and right parts of the equation on $\mathbf{v}$. Then, the auxiliary expression follows 


$$
\frac{\mathrm{m}(\dot{\mathbf{v}} \mathbf{v})}{\left(1-\mathrm{v}^{2} / \mathrm{c}^{2}\right)^{3 / 2}}=(\mathbf{F v})
$$

Substituting (10) in the initial relativistic equation, it turns out the second law of Newton with the new force $\mathbf{F}^{\prime}$ (the expression at the right-hand side):

$$
\mathrm{m} \dot{\mathbf{v}}=\sqrt{1-\mathrm{v}^{2} / \mathrm{c}^{2}}\left(\mathbf{F}-\mathbf{v}(\mathbf{F} \mathbf{v}) / \mathrm{c}^{2}\right)
$$

Students and postgraduates are well familiar with mechanics and hydrodynamics when in the most no relativistic area of speeds, forces appear depending on the speed of body movement (the concept of the attached mass etc., can be entered). If this is so, how do students can understand the importance of new relativistic dependences of force on velocity? Formally, there can be any force in the given expression of force $\mathbf{F}^{\prime}$ as a letter $\mathbf{F}$. However, the SRT is substantiated from the properties of light. Unfortunately, there are no proofs that the relativistic equation of movement can be applied to something, except to the charged particles being under an action of the Lorentz force. Let us remind ourselves that during the different time periods the Lorentz force was not the unique form for an electromagnetic force. Among the well-known expressions there were: Ampere's force, Weber's force and other ones. If the modern electrodynamics had the self-consistent character (because fields are manifested on their power influence), then the expression for electromagnetic force should be deduced from the Maxwell equations, instead of being artificially entered. Such an expression has been received in (Smulsky, 2004), and it differs from the expression of the Lorentz force. Incidentally, the experiments that were interpreted as proofs of the reality of relativistic length contraction and time dilation have a simple alternative interpretation (Jefimenko, 1998) in terms of velocity-dependent forces present in the systems.

Students operate well with limiting transitions. They see that the limiting transition from relativistic mechanics to classical mechanics does not exist (for some values there is not even an approximate transition!). Thus, the limiting transition from the Lorentz transformations to the Galileo transformations for the time $\left(t=t^{\prime}+v x^{\prime} / c^{2}\right)$ shows that the Newtonian mechanics is not simply a limit of low speed $\mathrm{v} / \mathrm{c} \ll 1$, but what is required is a quite different condition $\mathrm{c} \rightarrow \infty$, but the finite speed of light was defined in classical physics in the 17 th century!

Students disclose some oddities of the SRT, which in principle cannot be coupled with classical physics (regardless of the speed of movement). Newtonian space possesses an important property: systems with lower dimensions can possess similar properties. For example, the vector can be introduced not only in space but also on the line and the plane. At the SRT spatial values do not possess vector properties (only 4-vector), i.e. there is no continuous limiting transition to classical quantities ("nearly vector" $\rightarrow$ vector). 4-speed is always orthogonal to the 4-acceleration. 4speed of light is infinite. Here it may also be mentioned the non-commutativity of the relativistic velocity addition law for non-collinear vectors (fundamental difference).

The limiting transition to the classical energy is also problematic. We mentioned earlier about the condition of such a transition $\mathrm{c} \rightarrow \infty$. But then, not only the energy of rest, but also any energy becomes $E=\infty$. The limiting transition to low velocities for many variables raises a number of questions. All formulas should pass to the Newtonian form, when the rate of transmission of interactions is supposed infinite (e.g., Lagrange function, action, energy, the Hamiltonian function, and others.). However, we see that it is not so: 4-velocity goes over in a set of four numbers $(1,0,0,0)$ and does not mean anything, the 4-acceleration - also; the interval $S \rightarrow \infty$; the components of 4power tend to zero set, etc. How to explain to students an independent physical meaning of all these relativistic quantities and expressions?

Detailed coverage of the history with the transverse Doppler effect and the calculation of the value of effect are given in (Akimov, 2001). Here we present another method for derivation of the classic result that is understandable even to schoolchildren. The students are well aware of the difference between the pinhole source of light (that are spherical waves!) and the plane-parallel waves. For example, at the level of middle school one can strictly find an exact solution of the following problem: an oscillating up and down float excites circular waves on the water surface; how many peaks of waves per unit of time will be fixed by an observer moving along a line over the water surface? It is obvious that for plane waves no transverse Doppler effect exists. But if a student does not understand the difference between spherical and plane waves, then, apparently, he does not understand Physics or Mathematics. 
In the beginning we will consider the spherical waves generated by a moving dot source in the environment (for example, it can be a sound or circles on water). Let a rest receiver of signals is placed at the point $R$. If a source was based at the point $\mathrm{O}$ all the time, the direction of the signal distribution would be represented by a line RO (the length of a wave can be defined, if distance $|\mathrm{OR}|$ is divided into the number of accomplished oscillations in the time of passage of the given distance). The similar situation would be for a source which is based at some other point $\mathrm{j}$. Let now the source move rectilinearly with a constant speed $\mathbf{v}$. Let us choose conditionally a segment of length equals to the wavelength as an investigated signal and agree that we will see a point corresponding to the beginning of this signal (for uniform movement it is quite equivalent, it would be possible to see the movement of the middle or of the end of this conditionally allocated segment). The source was at point $\mathrm{O}$ at the moment of the beginning of the signal emission, and the source is at the point $j$ at the moment of the beginning of the reception of the same signal by the receiver (see Fig. 10). An angle $\theta$ is "the angle between velocity and the supervision line, measured in the receiver system" as it is usually in the theory of the Doppler effect. It is easy to define the change of a period of perceived oscillations $\mathrm{T}^{\prime}$ in comparison with the period $\mathrm{T}$ of oscillations for the source based at a point $\mathrm{O}-$ from interrelation of the sides of a triangle (distances, or, wavelengths - if the length of each side is divided into number $\mathrm{N}$ of the oscillations made during this time, where $\left.\mathrm{T}=\mathrm{t} / \mathrm{N}, \mathrm{T}^{\prime}=\mathrm{t}^{\prime} / \mathrm{N}\right)$. For this purpose we will take the theorem of cosines:

$$
\left.(\mathrm{ct})^{2}=(\mathrm{vt})^{2}+(\mathrm{ct})^{\prime}-2(\mathrm{ct})^{\prime}\right)(\mathrm{vt}) \cos (\pi-\theta) .
$$

Solving this quadratic equation for $t^{\prime}$, we have $t^{\prime}=t\left(\sqrt{1-\beta^{2} \sin ^{2} \theta}-\beta \cos \theta\right)$, where as usually $\beta=v / c$. As a result, for the frequency shift we receive the following expression:

$$
v^{\prime}=\frac{v}{\sqrt{1-\beta^{2} \sin ^{2} \theta}-\beta \cos \theta} .
$$

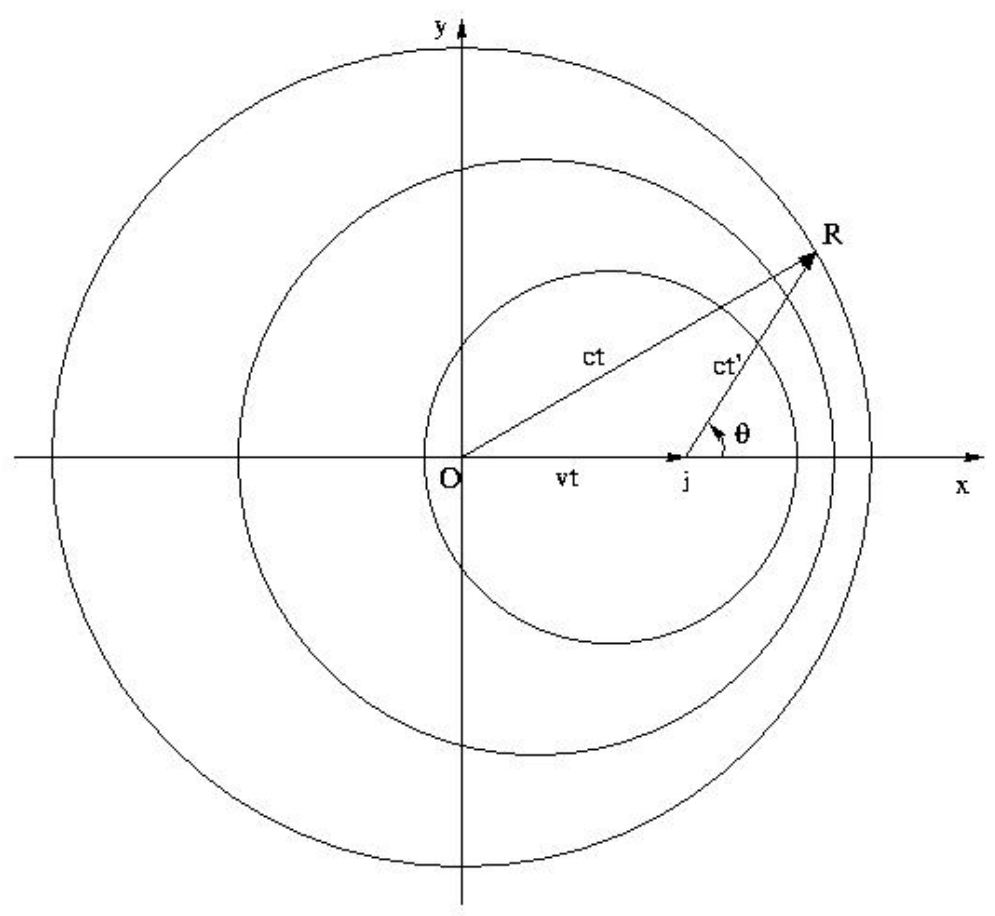

Fig. 10. The Doppler effect with a moving source

Let now spherical waves are generated in the environment by a quiescent source $\mathrm{j}$, and the receiver moves rectilinearly with a constant speed $\mathbf{v}$, and the latter is in the point $\mathrm{R}$ at the moment of the beginning of the signal 
reception. The situation to this moment is represented in Fig. 11. Similarly, using the theorem of cosines for the given triangle $\left(\mathrm{ct}^{\prime}\right)^{2}=\left(v t^{\prime}\right)^{2}+(\mathrm{ct})^{2}-2(\mathrm{ct})\left(\mathrm{vt} \mathrm{t}^{\prime}\right) \cos (\pi-\theta)$, and resolving the quadratic equation for $\mathrm{t}^{\prime}$, we find: $t^{\prime}=t\left(\sqrt{1-\beta^{2} \sin ^{2} \theta}-\beta \cos \theta\right) /\left(1-\beta^{2}\right)$. As a result the formula of the Doppler effect for spherical waves will look like:

$$
v^{\prime}=v\left(\sqrt{1-\beta^{2} \sin ^{2} \theta}-\beta \cos \theta\right)
$$

Its correctness at any distances follows from the procedure of derivation of the formula. Actually the angle $\theta$ also automatically tracks distance between a source and the receiver, since this angle varies in the course of movement, unlike a case of plane-parallel waves. First of all, in the received formula it is interesting for us the fact that there exists the transverse Doppler effect for spherical waves (if to substitute $\theta=\pi / 2$ in the last formula of the Doppler effect), completely coinciding with the relativistic expression. At simultaneous movement of the source and the receiver, the Doppler effect for spherical waves looks like:

$$
v^{\prime}=\frac{v\left(\sqrt{1-\beta_{1}^{2} \sin ^{2} \theta_{1}}-\beta_{1} \cos \theta_{1}\right)}{\sqrt{1-\beta_{2}^{2} \sin ^{2} \theta_{2}}-\beta_{2} \cos \theta_{2}} .
$$

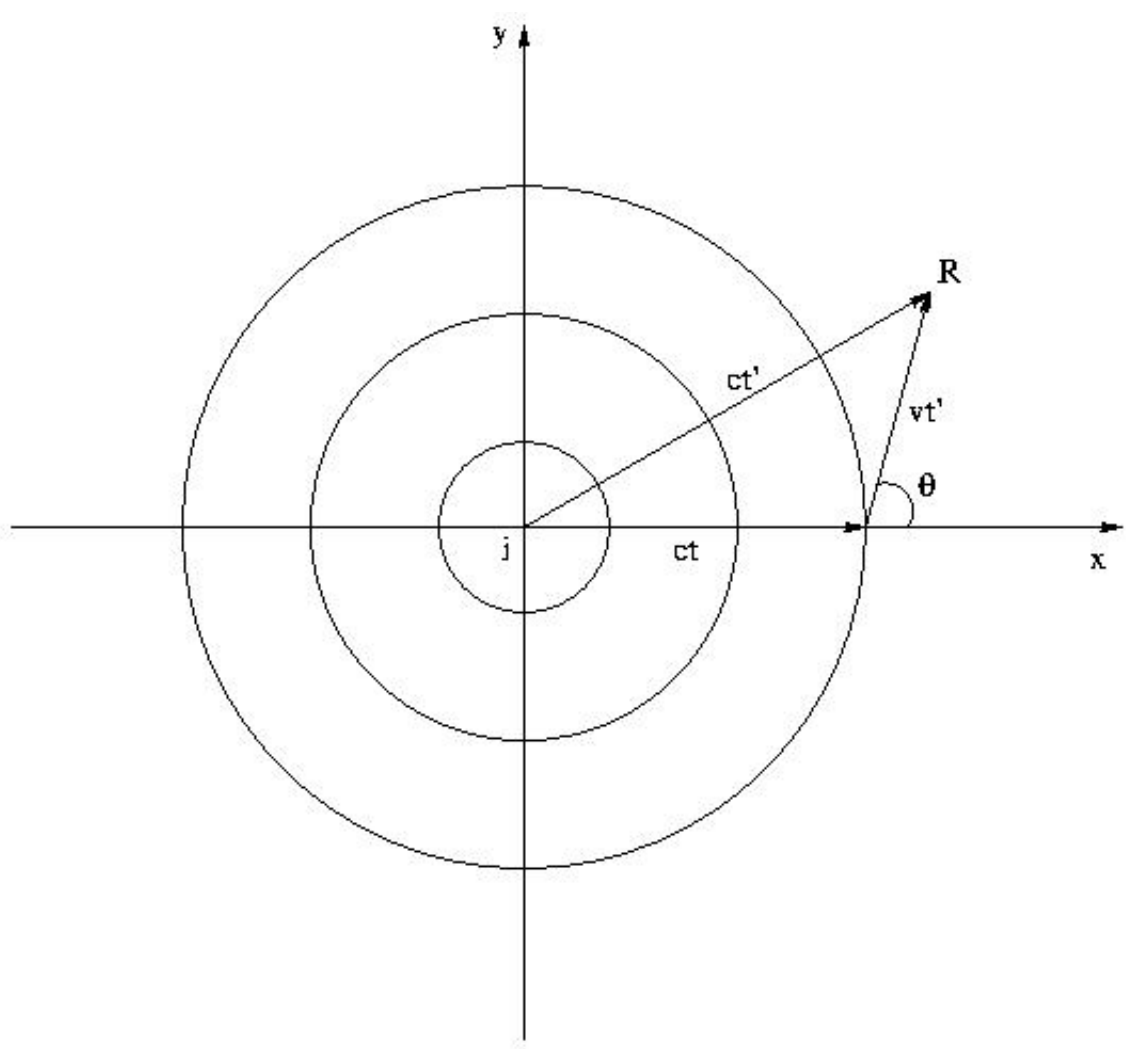

Fig. 11:- The Doppler effect with a moving receiver.

Max Laue was the last who wrote down one relativistic formula simultaneously including both movement of a source and a receiver. Then, it have been understood the contradiction of the simultaneous account of both movements to the relativistic ideology itself, but it did not be agreed upon which of the two formulas to discard (after all, A. Einstein used both!). As a result in the literature, different authors use different formulas. Besides it is not clear, how one and the same relativistic formula of the Doppler effect can simultaneously give two classical formulas of the Doppler effect at limiting transition (after all, both of them are experimentally checked up and give the different observed results, for example, for sound)? Which of versions to choose to teach students? 


\section{Discussion and conclusion:-}

Consideration of the work of electromagnetic clocks indicates that the time dilation is not a kinematic effect (Jefimenko, 1996).

Let us try to clear up why there is disagreement between the relativistic law of velocity addition and the Lorentz transformations, in spite of the fact that the first expression is derived from the second one in textbooks. Let us recall the derivation (for one-dimensional case): for mutual motion of systems $\mathrm{K}$ and $\mathrm{K}^{\prime}$, proceeding from the Lorentz transformation laws

$$
x_{1}=\frac{x+V t}{\sqrt{1-V^{2} / c^{2}}}, \quad t_{1}=\frac{t+x V / c^{2}}{\sqrt{1-V^{2} / c^{2}}}
$$

we divide the differential $\mathrm{dx}_{1}$ by $\mathrm{dt}_{1}$ with regard to definitions $\mathrm{v}=\mathrm{dx} / \mathrm{dt}$ and $\mathrm{v}_{1}=\mathrm{dx}_{1} / \mathrm{dt}_{1}$ and obtain:

$$
\mathrm{v}_{1}=\frac{\mathrm{v}+\mathrm{V}}{1+\mathrm{Vv} / \mathrm{c}^{2}} .
$$

This indicates the following things:

1. The observer is placed at the origin of system $\mathrm{K}$ and measures the distance $\mathrm{x}$ to the studied body in its system $\mathrm{K}$.

2. He considers time $\mathrm{t}$ to be universal in his system and determines the velocity of the body in his system $\mathrm{v}=$ $\mathrm{dx} / \mathrm{dt}$.

3. Using his own (!) time $\mathrm{t}$, he measures speed $-\mathrm{V}$ of system $\mathrm{K}^{\prime}$ with respect to $\mathrm{K}$ and considers the relative velocities of systems to be mutually opposite in direction. This observer cannot measure any other thing: the summary velocity $\mathrm{v}_{1}$ is a computable quantity.

In the essence of the formula, we cannot simply pass to the second substitution for determining $v_{2}$, though, formally, any arbitrary number of velocity values can be sequentially substituted into the expression for the relativistic law. In the case of addition of motions along a single straight line, the classical property of commutativity is conserved, and the contradiction is veiled. But we can apply another approach: we shall search for the sequence of three transformations of velocities that retains the initial time in the Lorentz transformation laws invariant. Then it can easily be verified that, instead of (5), a unique succession can be taken:

$$
\mathrm{v}_{1} \mathbf{i}, \quad \mathrm{v}_{2} \mathbf{j}, \quad-\mathrm{v}_{1} \mathbf{i} \sqrt{1-\mathrm{v}_{1}^{2} / \mathrm{c}^{2}}-\mathrm{v}_{2} \mathbf{j} .
$$

However, at first, the turning of segments remains. Second, a new set of velocities does not satisfy, in the given succession, the law of velocity addition, i.e., factually, there changes the order of substitution of the velocities $\mathrm{v}_{1}$ and $v_{2}$ (that is inconsistent with the essence of this law). Therefore, the difficulties are not eliminated in this case either. How this moment can be understood by students?

The next phrase is inconclusive for students: that "the SRT is simply a new geometry and, therefore, is noncontradictory". Students understand very well that Physics studies causes of phenomena and concrete mechanisms directly influencing on the phenomenon under investigation. Of course, to obtain mathematical solutions, different transformations of coordinates are frequently used in Physics (conformal ones, for example). As a matter of fact, it is elementary substitutions only. Nobody claims that the real change of the whole Universe from the outer region into the inner one of a circle follows from correctness of some solutions. Students guess that the existence of the Lorentz transformations in the SRT does not prove the objectivity of kinematic effects. First, the Lorentz transformations are not the only invariant, but only one of mathematical invariants of the wave equation. For example, the Fought transformations, which are also an invariant of the wave equation, have been discovered formerly. Secondly, from Mathematics itself no physical principles follow: the invariance property is completely defined by a combination of operations and "letters" in the equation. In particular, the Lorentz transformations with the speed of sound instead of the speed of light $\mathrm{c}$ can be used for some acoustic problems just because they are invariant. Thirdly, the Lorentz transformations are obtained for the process of light propagation in emptiness. But this is a particular physical phenomenon, and it is not necessary to exaggerate its generality. Let us notice that if some mathematical equation 
appears to be invariant as relative to the Lorentz-type transformations with some constant $c$, it means that among particular solutions of the given equation there are "surfaces" of the wave type, capable to extend with speed c. For mathematics not all- mathematical conclusions follow from the fact of invariance. Students do not understand the need to clone the only one unique invariance on properties of the whole world. After all, we do not make general conclusions from the invariants of the heat conductivity equation for hydrogen or from properties of hydrogen plasma only on the ground that all atoms consist of electrons, and kernels have protons. Students see that the Universe's symmetry does not coincide with the primitive spherical symmetry of dot light flashes in emptiness (look at crystals, at the live beings, at Space). Using one scalar constant c, it is impossible to define even speed of light in real substances (concrete gases, liquids, crystals), not to mention that, in general, perturbations in the environment extend with the speed of a sound. This last speed is not determined by one constant either, but depends on concrete substance (it is anisotropic in crystals, for example). All above mentioned facts are well known to students. Apparently, in general, it is impossible to adjust all properties of the world to one scheme of invariance. Except "properties" of emptiness, elements of environment (atoms and molecules possessing a huge variety of properties) are involved in processes even for processes of light distribution, also, interaction is involved with devices, which individualizes the processes at once (i.e., there should be at least something average between properties of emptiness and properties of concrete substance).

Students assume that actions of the SRT in kinematics can be called "obtaining images using flashes of light". It is known that images can be enlarged, reduced, distorted and false (in a curved mirror). But in any branch of Physics, besides the SRT, on the basis of such images the conclusion about the change of properties of the objects themselves is not made. On the contrary, a way to calibrate to extract the real information was searched. How can we convince the students that this case - special?

Obvious examples of the incompleteness of only relative values are present in classical mechanics. Contrary to the key idea of the isolation of systems for the application of the concept of "relativity" to them, the exchange of signals between the systems is used in the SRT. This raises numerous questions among students. In addition to the classic examples of differences between open and closed systems (in the hold or on the deck of a ship), there are differences to the process of establishing solutions. For example, the start of motion of a frame in the magnetic field causes an instantaneous emergence of a current in it, but the movement of a magnet leads to the emergence of current some time later. Further, the presence of any dynamic characteristics (in addition to the kinematic ones) immediately individualizes the process. Consider an elementary mechanical example: Let some small ball drop to the Earth nonelastically. Define kinetic energy, which was transformed into heat. Relative velocity is one and the same for the ball and for Earth. Why do we substitute into the formula, the mass of the ball but not the mass of the planet Earth? These examples demonstrate that locally absolute velocities often play a role (using the relative velocity, one can sometimes get an approximate answer).

The authors fully share the opinion of R. Feynman that the existing problems must not be covered up under the carpet, especially when we talk about the education of students, in order not to spoil the young souls. That is why these issues should be discussed by the wider scientific and educational community.

\section{References:-}

1. Akimov, O. E. (2001). Natural Sciences, (Unity Dana, Moscow).

2. Einstein, A. (1905). Zur Elektrodynamik bewegter Körper. Ann. Phys., 17: 891-921.

3. Frankfurt, U.I. and Frank, A.M. (1972). Optika Dvizhushchikhsya Tel (Optics of Moving Bodies, Moscow: Nauka). (in Russian).

4. Jefimenko, O. D. (1998). On the Experimental Proofs of Relativistic Length Contraction and Time Dilation. Zeitschrift für Naturforschung 53a: 977-982.

5. Jefimenko, O. D. (1996). Direct Calculation of Time Dilation. American Journal of Physics 64(6): 812-814.

6. List of some antirelativistic works (1905-2003) in http://www.antidogma.ru/library/index_en.html .

7. Poincare, H. (1983). On Science, (Nauka, Moscow). (in Russian).

8. Smulsky, J.J. (2004). The Theory of Interaction (Publishing house "Cultural Information Bank", Ekaterinburg). 